\title{
SARS-CoV-2 mRNA vaccine BNT162b2 elicited a robust humoral and cellular response against SARS- CoV-2 variants.
}

Daniele Lilleri ( $\sim$ D.Lilleri@smatteo.pv.it)

Fondazione IRCCS Policlinico San Matteo

Irene Cassaniti

Molecular Virology Unit, Microbiology and Virology Department, Fondazione IRCCS Policlinico San

Matteo, Pavia. Italy

Federica Bergami

Fondaz. IRCCS Pol. San Matteo

Elena Percivalle

Fondazione IRCCS Policlinico San Matteo

Elisa Gabanti

Fondaz. IRCCS Pol. San Matteo https://orcid.org/0000-0003-4865-4006

Josè Camilla Sammartino

Fondaz. IRCCS Pol. San Matteo

Alessandro Ferrari

Fondaz. IRCCS Pol. San Matteo

Kodjo Adzasehoun

Fondaz. IRCCS Pol. San Matteo

Federica Zavaglio

Fondaz. IRCCS Pol. San Matteo

Paola Zelini

Fondaz. IRCCS Pol. San Matteo

Giuditta Comolli

Fondaz. IRCCS Pol. San Matteo

Antonella Sarasini

Fondaz. IRCCS Pol. San Matteo

Antonio Piralla

Fondaz. IRCCS Pol. San Matteo

Alessandra Ricciardi

Fondaz. IRCCS Pol. San Matteo

Valentina Zuccaro

Fondaz. IRCCS Pol. San Matteo 


\section{Fabrizio Maggi}

ASST Sette Laghi, Università dell'Insubria

\section{Federica Novazzi}

ASST Sette Laghi

\section{Luca Simonelli}

Institute for Research in Biomedicine

\section{Luca Varani}

Institute for Research in Biomedicine (IRB), Università della Svizzera italiana (USI) https://orcid.org/0000-0002-0963-0987

\section{Fausto Baldanti}

Fondazione IRCCS Policlinico San Matteo

\section{Article}

Keywords: vaccines, SARS-CoV-2, antibodies

Posted Date: April 9th, 2021

DOI: https://doi.org/10.21203/rs.3.rs-396284/v1

License: (c) (i) This work is licensed under a Creative Commons Attribution 4.0 International License. Read Full License 


\section{Abstract}

BNT162b2 vaccine was introduced in Italy on 27th December 2020 and healthcare workers were rapidly vaccinated. In this study, we demonstrated that one vaccine dose was sufficient for eliciting a sustained humoral and cell-mediated response in SARS-CoV-2 experienced healthcare workers but had a lower effect in SARS-CoV-2 naïve subjects. However, $98 \%$ naïve subjects developed both neutralizing antibodies and Spike-specific T-cells after the second dose. Moreover, the antibody and T-cell responses were effective against viral variants since a partial reduction in antibody response was observed only against the South-African variant in SARS-CoV-2 naïve individuals, while the T-cell response was less affected.

\section{Introduction}

After the emergence of the Coronavirus Disease 2019 (COVID-19) pandemic caused by the betacoronavirus named severe acute respiratory syndrome virus 2 (SARS-CoV-2), a strong effort has been made for the rapid development of protective vaccines. The BNT162b2 vaccine [1], a lipid nanoparticleformulated, nucleoside-modified RNA encoding the SARS-CoV-2 full-length Spike (S) protein derived from the first SARS-CoV-2 isolated in Wuhan, China, modified by two proline mutations to lock it in the prefusion conformation, was the first authorized for active immunization.

The vaccine showed a 95\% protection against SARS-CoV-2 infection in a phase $2 / 3$ trial [2]. Another mRNA-based vaccine, mRNA-1273 [3] showed similar effect and was subsequently authorized. However, data on the kinetics of immune response elicited by the vaccines are limited to low numbers of subjects analyzed and mainly to the antibody response $[1,4-8]$.

Moreover, the T-cell response elicited by the vaccine may have a crucial role in the protection from SARSCoV-2 infection and disease as well as in the establishment of a long-term recall memory response. Previous reports related to SARS-CoV-1 infection suggested a protective role of both humoral and cellmediated immunity. Moreover, while SARS-CoV-1 T-cell response may confer long-term protection, the antibody response was found to be relatively short-lived in convalescent individuals [9]. On the other hand, T- and B-cell memory specific for SARS-CoV-2 was found to persist for at least 6-8 months [1012].

The emergence of new SARS-CoV-2 variants with mutations in the Spike protein has raising significant concerns about vaccines efficacy and reinfection risk in subjects who experienced a natural SARS-CoV-2 infection. The first emerging spike protein variant, D614G, rapidly became the dominant SARS-CoV-2 clade in Europe by May 2020 (EU variant). Recently, the new variant 501Y.V1 lineage B.1.1.7 (UK variant) including multiple mutations in both the receptor binding domain (RBD) and $\mathrm{N}$-terminal domain of $\mathrm{S}$ protein [13] as well as the 501Y.V3 lineage P.1 (Brazilian, BZ) [14] and 501Y.V2 lineage B.1.351 (South African, SAF) variants mutations are present in S protein and, especially, in the RBD [15].

Preliminary data suggested that convalescent and sera from vaccinated individuals efficiently neutralized UK variant, while a reduction in neutralizing (NT) antibody titres has been observed against SAF variant 
$[16,17]$

In addition, recent data from adenoviral vaccine ChAd0x1 nCoV-19 studies reported a lower efficacy against SAF variant [18], whereas the Ad26.COV2.S vaccine appeared equally effective in USA, Brazil and South Africa, where different variants circulate [19]. The question of whether both SARS-CoV-2 experienced patients and vaccinated subjects might be protected against emerging variants needs to be urgently investigated. So far, in depth analysis of the level of vaccine-induced B- and T-cell immune response against SARS-CoV-2 variants in vaccinated subjects is still lacking.

The present study was designed to evaluate the development and the persistence of the T- and B-cell responses in subjects receiving the BNT162b2 vaccine. In this article, we report the analysis of the immune response in 145 subjects after the first and second dose of the vaccination schedule, whereas the long-term persistence of the immune response will be analyzed subsequently. Moreover, we aimed to define the level of both antibody and cell mediated response against the emerging SARS-CoV-2 variants after a complete vaccination schedule in comparison to convalescent patients.

\section{Results}

\section{Subjects enrolled}

The immune response to the mRNA vaccine BNT162b2 was evaluated in 145 healthy healthcare workers receiving vaccination between December 27, 2020 and February 11, 2021. Among the subjects enrolled in the study, 127 (87.6\%) were SARS-CoV-2 naïve (30 males and 97 females) and 18 (12.4\%) SARS-CoV-2 experienced (1 male and 17 females) before vaccination. The median age was 44 (range 21-69) years.

\section{Antibody response in SARS-CoV-2 naive and experienced vaccinated subjects}

After the first dose (T1, day 21) all the 18 SARS-CoV-2 experienced subjects showed anti-RBD antibody level above the upper limit of the quantifiable range of the assay (2500 arbitrary units $[\mathrm{U}] / \mathrm{mL}$ ), while 122/127 (96.1\%) SARS-CoV-2 naïve subjects developed a detectable anti-RBD antibody response, although at significantly lower levels (median $43.5 \mathrm{U} / \mathrm{mL}$; IQR 16.1-95.7 U/mL) than experienced subjects did. Moreover, levels of anti-RBD antibodies in naïve subjects at T1 were significantly lower ( $p=$ 0.009 ) than baseline levels of experienced subjects (median $88.2 \mathrm{U} / \mathrm{mL}$; IQR $45-203.9 \mathrm{U} / \mathrm{mL}$ ).

After the second dose (T2, day 42), all the SARS-CoV-2 naïve subjects developed a positive anti-RBD response; however, the median anti-RBD level was still significantly higher in SARS-CoV-2 experienced (all the subjects developed anti-RBD level higher than $2500 \mathrm{U} / \mathrm{mL}$ ) than in SARS-CoV-2 naïve subjects (median 1803 IQR1002- $\geq 2500 \mathrm{U} / \mathrm{mL} ; \mathrm{p}<0.001$ ). (Fig. 1a)

A similar trend was observed for NT antibodies: all SARS-CoV-2 experienced subjects were positive at T1, showing NT titres at the upper limit of the assay $(\geq 1: 640)$ in all but three subjects, in whom NT titres increased to $\geq 1: 640$ after the second dose (T2). On the other hand, 119/127 (93.7\%) SARS-CoV-2 naïve subjects developed detectable SARS-CoV-2 NT antibodies at T1 (median 1:40; IQR 1:20-1:160), and NT 
titers increased at T2 (median 320; IQR 1:320- $\geq 1: 640$ ), becoming detectable in all subjects (Fig. 1b). Serum NT titres were significantly higher in SARS-CoV-2 experienced than in naïve subjects at any time point.

A weak inverse correlation between age and anti-RBD level was found at both T1 and T2 in SARS-CoV-2 naïve subjects, although only at T1 was statistically significant $(r=-0.33$; IC95\% -0.48 to $-0.16 ; p<0.001$ and $r=-0.16$; IC95\% -0.33 to $0.02 ; p=0.068$, respectively). Similarly, a weak inverse correlation was observed between age and NT antibodies at T1 $(r=-0.40$; IC95\% -0.54 to $-0.23 p<0.001)$, and no significant correlation was observed at T2 $(r=0.02 ; \mathrm{IC} 95 \%-0.16$ to $0.20 p=0.829)$.

\section{SARS-CoV-2 specific T-cell response}

ELISpot assay was used for detecting anti-S and, as control, anti-nucleocapsid (N) cell-mediated response in vaccinated subjects at baseline, at T1 and T2.

All but one SARS-CoV-2 experienced subjects (17/18, 94.4\%) showed S-specific T cells at baseline (median 27.50 IFNy SFU/million PBMC; IQR 13.8-52.5), and levels of S-specific T cells increased at T1 (median 80.0 IFNY SFU/million PBMC; IQR 47.5-315; $p<0.001$ ) and did not further increase at T2 (median 172.5 IFNy SFU/million PBMC; IQR 85-255; $p=0.510$ ).

Focusing on SARS-CoV-2 naive subjects, the percentage of responders was $69.3 \%$ at T1 (median level 20 IFNy SFU/million PBMC; IQR 5-57.50) and $98.4 \%$ at T2 (median level 110 IFNy SFU/million PBMC; IQR 60192.5). Levels of S-specific T cells significantly increased from T1 to T2 $(p<0.001)$; even if the level of Sspecific T-cells at T2 in experienced subjects was higher than that observed in naïve subjects at the same time point, the difference was not statistically significant $(p=0.095)$. No significant correlation was observed between age and Spike specific T-cell response at both T1 ( $r=-0.02$ IC95\% $-0.21-0.17 ; p=0.8214)$ and T2 ( $r=-0.17$; IC95\% -0.32-0.04; $p=0.107)$.

Levels of N-specific T cells did not change with time in both experienced and naïve subjects (Fig. 2b). Of note, $30 \%$ of SARS-CoV-2 naïve subjects showed detectable SARS-CoV-2 specific T-cell response against $\mathrm{S}$ and $18.2 \%$ against $\mathrm{N}$ at baseline.

The phenotype of S-specific T cells elicited by the vaccine was analysed in a subgroup of 20 naïve subjects at $\mathrm{T} 2$ by flow-cytometry analysis of T-cells proliferating in response to $\mathrm{S}$ and $\mathrm{N}$ peptide pools and co-expressing CD25 and ICOS activation markers (Fig. 3; Supplementary Fig. 2). The vaccine elicited

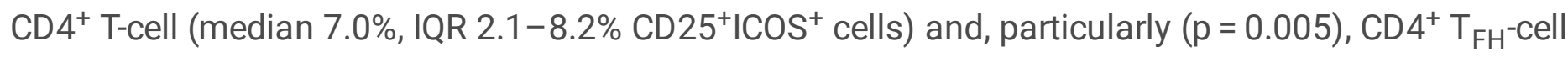

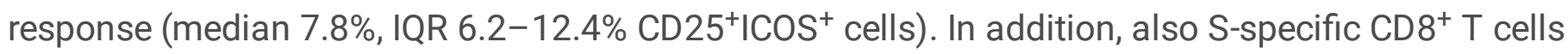
(median 2.6\%, IQR 1.4-6.1\% $\mathrm{CD}^{-} 5^{+} \mathrm{ICOS}^{+}$cells) were detected in vaccinated subjects. This pattern is similar to that observed in 9 SARS-CoV-2-infected subjects with mild symptoms collected a median time of 38 (range 28-90) days after infection, who showed a slightly lower number of proliferating $\mathrm{CD} 4^{+} \mathrm{T}$ and $C D 4^{+} T_{F H}$ cells than vaccinated subjects ( $p=0.044$ and 0.047 , respectively). As observed also with the ELISpot assay, the response to $\mathrm{N}$ protein was detected in few vaccinated subjects. 
Overall, after the first dose, the overall percentage of full responders (i.e. subjects developing both $T$ cells specific for SARS-CoV2 S protein and NT antibody) was $77.8 \%$ (95\% Cl: 70.1-84.0), corresponding to $100 \%$ of experienced and $68.8 \%$ (95\% Cl: $60.2-76.3$ ) of naïve subjects. After the complete vaccination schedule, the overall percentage of full responders was $98.6 \%$ (95\% Cl: $95.0-99.8$ ), corresponding to $100 \%$ of experienced and $98.4 \%$ (95\% Cl: $94.4-99.7)$ of naïve subjects.

\section{Correlation between T-cell response, binding antibodies and neutralizing antibodies}

Anti-RBD antibody level and SARS-CoV-2 NT antibodies were strongly correlated at T1 ( $r=0.74$ IC95\% $0.66-0.81 ; p<0.001$; Supplementary Fig. $1 a)$ while S-specific T-cell response was poorly correlated with NT antibodies ( $r=0.30$, IC95\% 0.15-0.45, $p<0.001$; Supplementary Fig. $1 \mathrm{~b})$ and anti-S antibodies $(r=$ 0.41, IC95\% 0.26-0.54, p<0.001; Supplementary Fig. 1c). Correlation between SARS-CoV-2 NT and antiRBD antibodies was still significant but low at T2 $(r=0.34$, IC95\% 0.18-0.47, $p<0.001$; Supplementary Fig. 1d). On the other side, at T2 correlation between S-specific T-cell response and NT or anti-RBD antibody levels was not statistically significant $(r=0.16$, IC95\% $-0.01-0.31, p=0.060$ and $r=0.13, I C 95 \%$ $-0.04-0.29, p=0.13$, respectively) (Supplementary Fig. 1e-f).

\section{Change in lymphocyte counts}

Total $C D 4^{+}$and $C D 8^{+} T$ cells declined significantly $(p<0.001)$ at $T 1$, moving from a baseline mean count $( \pm$ SD) of $1042( \pm 371)$ to $928( \pm 331)$ cells/ $\mu$ l and from $543( \pm 221)$ to $468( \pm 185)$ cells/ $/$ l, respectively. At $\mathrm{T} 2$, mean levels of $\mathrm{CD} 4^{+}(952 \pm 359)$ and $\mathrm{CD} 8^{+}(474 \pm 204)$ T cells did not change significantly from T1, remaining lower than baseline. Total B cells did not change significantly from baseline $(275 \pm 143)$ to T1 $(260 \pm 190)$, and were slightly but significantly $(p<0.001)$ reduced at T2 $(248 \pm 121)$. Total NK cells did not change significantly after vaccination.

\section{Antibody and T-cell response against SARS-CoV-2 variants}

Sera collected at T2 from 31 subjects for SARS-CoV-2 infection were tested against RBD of the wild type (WT) Chinese strain, the 501Y.V1 lineage B.1.1.7 variant (UK) and 501Y.V2 lineage B.1.351 variant (South African; SAF) strains. RBD from WT and European (EU) D614G variant strains are similar, since the D614G mutation is located on the stem of the $S$ protein. Overall, median RBD-specific reciprocal antibody titres were 5838 [IQR 2675-16351] for WT (and EU) strain and 3220 [801-9263] and 60.5 [<50-196] for UK and SAF strains, respectively (Fig. 4a). A reduction of approximatively $50 \%$ and $99 \%$ of RBD-specific antibody titres was observed from WT to UK and SAF strains, respectively (Fig. 4b-c).

Sera collected at T2 from 68 SARS-CoV-2 vaccinated subjects (61 naïve per COVID-19 and 7 COVID-19 positive) were tested against WT strain and challenged against EU, UK, SAF, and 501Y.V3 lineage P.1 (Brazilian; BZ) (Fig. 4d). Among the 61 SARS-CoV-2 naïve subjects, an increase in four-fold dilution for SARS-CoV-2 NT antibodies was observed against the EU variant with respect to WT strain $(p<0.001)$

(Fig. 4e), while the level of SARS-CoV-2 NT antibodies was not affected when sera were tested against UK and BZ strains (Fig. 4f-g). A five-fold and twenty-fold reduction in median titre of SARS-CoV-2 NT antibodies was observed in response to SAF strain in comparison to WT virus and EU strain, respectively 
$(p<0.001)$ (Fig. 4h). However, although at lower levels NT antibodies against the SAF variant were still detectable in in 55/68 (80.9\%) subjects.

Among the 7 SARS-CoV-2 experienced vaccinated subjects, a sustained NT level against the all five variants was observed. The median reduction of SARS-CoV-2 NT against the SAF variant was only onefold than respect to WT (median 160 vs 320; respectively $p=0.603$ ).

In addition, sera from six vaccinates subjects, were used to assess the NT level also against the C.36 Thailand (Thai) and B.1.525 Nigerian (NIG) variants. No significant difference was observed between SARS-CoV-2 NT titer against WT, UK, BZ and Thai variant $(p=0.999)$. Similarly to what observed for the SAF variant, a six-fold decrease of NT level was documented against the NIG variant $(p=0.081$; Supplementary Fig. 3).

Poor correlation between RBD-specific and NT antibody titres for the different SARS-CoV-2 variants was observed. In 36 of the 68 vaccinated subjects (53\%), residual PBMC isolated at T2 were challenged against inactivated virus preparations from supernatant of Vero E6 cells infected with the five SARS-CoV2 strains (WT, EU, UK, BZ and SAF) and cell-mediated response was analysed by ELISpot assay (Fig. 6a). The ELISpot assay was less sensitive in measuring T-cell response using WT virus than using peptide pools from the homologous S protein: median IFNy SFU/million PBMC were 112.5 (IQR 71.3-233.8) and 47.5 (IQR 23-91), respectively. However, even if a slightly lower level of cell-mediated T-cell response was observed against UK, BZ and SAF variants (Fig. 6a), SARS-CoV-2 specific T cells were detected in the large majority of the subjects, with no significant difference in the frequency of responders. In detail, 34/36 (94.4\%) were positive for WT SARS-CoV-2 T-cell response and 33/36 (91.7\%) were positive for EU SARS-CoV-2 T-cell response. UK, BZ and SAF-specific T-cell response was detectable in 30/36 (83.3\%), $29 / 36(80.5 \%)$ and $31 / 36(86.1 \%$ ) vaccinated subjects (Fig. 6b), indicating that the BNT162b2 vaccine was able to elicit T-cells able to recognize conserved epitopes from any virus variant tested.

\section{Discussion}

Here we report a prospective and comprehensive evaluation of the development of antibody and T-cell responses elicited by the mRNA vaccine BNT162b2 for SARS-CoV-2 in a cohort of 145 immunocompetent individuals (18 SARS-CoV-2 experienced and 127 SARS-CoV-2 naïve). The major results of the study show that: i) all vaccinated subjects tested developed a NT antibody response, and $98.6 \%$ a specific T-cell response after complete vaccination schedule; both antibody and T-cell response were not affected by age after the second dose; ii) antibody and T-cell responses were not correlated; iii) the antibody and T-cell responses are effective against most viral variants (a reduction in antibody response was observed only against the SAF variant, while the T-cell response was less affected).

The mRNA-based vaccine was highly effective in eliciting both antibody and T-cell responses. Regarding the T-cell subsets induced, we observed both $\mathrm{CD} 4^{+}$and $\mathrm{CD} 8^{+} \mathrm{T}$ cells proliferative responses. This pattern is similar to that observed in a preclinical study for another mRNA-based vaccine against human 
cytomegalovirus [20]. Among $C D 4^{+} T$ cells, we observed an effective response of the $T_{F H}$ subset, which is involved in providing help for B-cell differentiation and antibody affinity maturation. A reduction in total T and $B$ cells after vaccination was observed; this event may be a consequence of lymphocyte redistribution to lymphoid organs during the development of the immune response to the vaccine.

In this study, we observed that all the SARS-CoV-2 naïve individuals developed a humoral response higher than that observed after natural infection after two vaccine doses but not after one vaccine dose, when about $6 \%$ of individuals was still negative for SARS-CoV-2 NT Abs. On the other side, the cell-mediated response was detectable in $98.4 \%$ naïve individuals after two doses of vaccine, whereas one vaccine dose was not sufficient for eliciting detectable SARS-CoV-2 T-cell response in $32.1 \%$ of subjects.

Conversely, both humoral and cellular responses were strongly induced after the first dose of the BNT162b2 vaccine in previously SARS-CoV-2 infected subjects: therefore, it appears that prime vaccine dose acts as a boost in these subjects. Moreover, both antibody and T-cell responses did not appear to increase after the second dose in these individuals. According to previous observation [21,22], this evidence strengthens the concept that just one vaccine dose may be sufficient in subjects with previous SARS-CoV-2 infection [23].

On the other side, we observed that level of antibody and, especially, cell-mediated response after one vaccine dose was weak or undetectable in SARS-CoV-2 naive subjects. This is in contrast to what was observed in a previous report analysing the antibody response in a small number of subjects [20], and suggests that only one dose could not be sufficient to elicit a complete protection in naive subjects. Furthermore, in these subjects the emergence of viral resistance or escape might be increased if a suboptimal immune response is elicited. Thus far, while a single vaccine dose may be sufficient in SARSCoV-2 experienced subjects, it can be unfavourable in SARS-CoV-2 naive subjects. Also, while the antibody levels observed after the first dose were inversely correlated with age, thus resulting in a potentially ineffective immunization in subjects at higher risk for developing the more severe consequences of SARS-CoV-2 infection, the second dose was effective in boosting the response in all subjects, with no correlation with their age.

Among the 5 SARS-CoV-2 variants tested, only SAF (501Y.V2 lineage B.1.351), significantly impacted neutralizing antibody levels in vaccinated subjects, while SARS-CoV-2 T-cell response was not significantly affected. Since the beginning of the pandemic, about 100 million individuals were infected by SARS-CoV-2 and neutralizing antibody titres are extremely variable [24]. Those with low titre could be re-infected, and due to the presence of incomplete protective neutralizing antibodies might develop escape variants $[25,26]$. The accumulation of mutations in RBD and N-terminal domain of Spike protein might be associated to increased escape from neutralization. Mutation at 484 site has been related to the reduction in neutralization sensitivity [27, 28]. Spike mutations in the South African 501Y.V2 lineage B1.351 are linked to immune escape from several classes of anti-SARS-CoV-2 monoclonal antibodies, and a significant decrease in neutralization titer of plasma from convalescent subjects was observed when sera were challenged with South African variant [29]. Usually, after natural infection or vaccination 
a polyclonal immune responses arise against multiple antigenic epitopes. Consequently, small numbers of variations in antigen sequence should have little impact on recognition by the immune system, including both NT antibodies and T cells.

Our results show that vaccinated individuals developed an equally effective NT antibodies response against the WT and UK variant, as well as against BZ variant, while the NT response against the EU variant was increased. On the other side, according to previous observation, sera from vaccinated subjects showed a significant reduction in SARS-CoV-2 NT antibodies when challenged against the SAF variant. Nevertheless, serum from the majority (almost $80 \%$ ) of vaccinated subjects maintained a neutralizing activity against this variant that could be effective in preventing the development of severe disease. These results are in contrast with another report showing a higher reduction in neutralization titres against pseudoviruses with S protein from UK, BZ and SAF variants, with respect to WT, and no increase in NT against European variant [30]. Although the binding titer to SAF RBD was highly reduced with respect to WT (about 100 folds), the NT titer was less affected, showing a five-fold reduction. In addition, we observed that anti-RBD antibody levels, measured in vaccinated subjects with either commercial or in house assays, have a poor correlation with NT titers. This may suggest that anti-S antibodies binding to sites outside the RBD, which are less involved by the mutations reported, could contribute to virus neutralization. Importantly, our studies rely on the use of wild type viruses rather than pseudoviruses. So far, the use of pseudoviruses could give discrepant results with respect to natural strains, as reported [31], likely because the artificial lentiviral particles cannot resemble the complete biology of actual clinical isolates.

Interestingly, the level of NT antibodies against SARS-CoV-2 variants in SARS-CoV-experienced vaccinated subjects was robust. This preliminary observation might be helpful in the design of vaccination strategies, suggesting that three exposures to SARS-CoV-2 Spike antigen could increase the level of neutralizing antibodies against SARS-CoV-2 variants.

However, and most considerably, our studies on cell-mediated response revealed that SARS-CoV-2 T-cell response is minimally affected by the mutations occurring in SARS-CoV-2 variants, as reported [32], and most subjects tested showed a detectable T-cell response against each virus strain. Differently from Tarke and colleagues, we used inactivated preparations of different SARS-CoV-2 variant isolates in order to recapitulate realistically antigen presenting mechanisms and natural epitope recognition from $T$ cells. The levels of T-cell response and NT antibodies elicited by the vaccine were not correlated; therefore, a low NT titer does not exclude the presence of a protective T-cell response able to control SARS-CoV-2 infection and avoid the development of a severe disease.

Furthermore, potential cross-reactive T-cells originally elicited by the common cold coronaviruses may be boosted by the vaccine and contribute to protection. In our study, about 20-30\% SARS-CoV-2 naïve subjects showed a T-cell response specific for $\mathrm{S}$ or $\mathrm{N}$ proteins. This might be related to the presence of cross-reactive epitopes from common cold coronaviruses that seasonally circulates [12, 33-36]. 
In conclusion, the BNT162b2 vaccine appears able to induce roust antibody and T-cell responses in all immunocompetent individuals and pave the way for the clinical evaluation of other mRNA vaccines currently in development [37]. Although the effectiveness of the antibody response may be partially reduced by some mutations naturally arising in virus strains, the T-cell response appears to be marginally affected. Although we analysed the T-cell response induced by BNT162b2 mRNA vaccine, it is likely the other SARS-CoV-2 mRNA vaccine licensed, the mRNA-1273 show a similar broadly reactive T-cell response. An important issue in the future evaluation of SARS-CoV-2 vaccines is the persistence of the memory response induced. This aspect will be prospectively addressed by our ongoing study.

\section{Methods}

\section{Study design}

We designed an observational, longitudinal prospective study to evaluate the immune response elicited by the BNT162b2 vaccine against SARS-CoV-2. The efficacy end-point were the development of SARSCoV-2-specific neutralizing antibody and T-cell response. The efficacy evaluation are conducted at shortterm (after the complete vaccination schedule), medium-term (6 months after vaccination); long-term (12 months after vaccination). Here we report results of the short-term evaluation.

Heparin-treated and untreated blood samples were collected before vaccination (T0), 21 days after the first dose (T1, before second dose administration) and 21 days after the second dose (T2). Blood lymphocyte counts, SARS-CoV-2 S-specific T-cells, RBD-binding antibody levels and SARS-CoV-2 NT serum titers were determined at each time point.

The primary end-point of the study was the frequency of "full responders" (i.e. subjects developing both T cells specific for SARS-CoV2 S protein and NT antibody) after the second dose of BNT162b2 vaccine. Secondary end-points were the following: i) the frequency of "full responders" after the first dose of BNT162b2 vaccine; ii) the magnitude of T cells specific for SARS-CoV2 S protein, RBD-binding and NT antibodies after the first and the second dose of BNT162b2 vaccine; iii) the correlation between T-cell response, RBD-binding antibodies and NT antibodies; iv) the changes in total CD4+ and CD8+ T cells, B cells and NK cells after the first and second dose of BNT162b2 vaccine; 5) the frequency of T-cell and NT antibody responders against SARS-CoV-2 variant strains; 6 ) the persistence of T-cell response, RBDbinding antibodies and NT antibodies. This last end-point will be analyzed at a later time.

Data about the frequency of subjects developing T cells and NT antibodies in response to the BNT162b2 vaccine were limited, thus this was an exploratory study. The phase $2 / 3$ clinical trial [2] showed a $95 \%$ (95\% Cl: $90-98 \%)$ efficacy of the vaccine in protection from SARS-CoV-2 infection. Thus, adopting a conservative approach, we hypothesized a $90 \%$ rate of "full responders" developing both T cells specific for S protein and NT antibody. With a sample size of 150 subjects, the $95 \%$ confidence interval of the expected frequency is $84-94 \%$. This correspond to a minimum number 126 "full responders" which should have been detected. 
The study (CoVax) was approved by the local Ethics Committee (Comitato Etico Area Pavia) and Institutional Review Board (P-20210000232). All the subjects signed informed written consent.

\section{Lymphocyte counts}

Total lymphocytes subpopulation count was determined on whole blood by flow cytometry using BD Lyric flow cytometer, (BD Biosciences, San Jose,CA, USA). After lysis of red blood cells, $\mathrm{CD} 3^{+} \mathrm{CD} 4^{+}$, and $\mathrm{CD}^{+} \mathrm{CD} 8^{+} \mathrm{T}$ cells, $\mathrm{CD} 16^{+} / 56^{+} \mathrm{NK}$ and $\mathrm{CD} 19^{+} \mathrm{B}$ cells/ $\mu$ l were determined by flow cytometry, using $\mathrm{BD}$ Multitest $^{\text {TM }} 6$-color TBNK reagent and TruCOUNT tubes (BD Biosciences). Gating strategy was set up on CD45 $5^{+}$and side scatter (SSC).

\section{SARS-COV-2 variants isolation and viral titration}

SARS-CoV-2 strains, including wild type China-derived strain (D614), European strain (D614G), UK strain (501Y.V1 lineage B.1.1.7), Brazilian strain (501Y.V3 lineage P.1) and South Africa strain (501Y.V2 lineage B.1.351) were isolated from infected patients' nasal swabs; in detail, $200 \mu \mathrm{l}$ of each sample was inoculated and propagated into Vero E6 (VERO C1008 (Vero 76, clone E6, Vero E6; ATCC1CRL-1586TM) permissive cell line and titrated to prepare cell free virus for neutralization assay. All the strains were sequenced in order to confirm the presence of variant-defining mutations. Complete genome sequencing was performed in order to confirm the presence of variant-defining mutations and sequences were submitted to GISAID under the following reference numbers (EPI_ISL_568579; EPI_ISL_1403609-11).

\section{RBD-ELISA}

Half-area 96-well microplates were coated for $1 \mathrm{~h}$ with $5 \mu \mathrm{g} / \mathrm{ml}$ recombinant RBDs [38] of the B1 (China/European) strain, B.1.1.7/N501Y.V1 (UK) strain, or the B.1.351/501Y.V2 (South African) strain. After overnight (or $1 \mathrm{~h}$ ) blocking with $5 \%$ (wt/vol) skimmed milk, the plates were washed and incubated for $1 \mathrm{~h}$ with human serum four fold serial dilutions (starting from 1:50), then for $45 \mathrm{~min}$ with horseradish peroxidase-labeled goat IgG to human IgG and, finally, for $25 \mathrm{~min}$ with $5 \mathrm{mg} / \mathrm{ml}$ orthophenylendiamine before the addition of $4 \mathrm{~N}$ sulphuric acid. The optical density (OD) value of the serum incubated without RBD was subtracted from the OD value of the serum incubated with RBD. Cutoff of 0.100 net OD was calculated based on mean+2SD results of SARS-CoV-2 naive subjects at the serum dilution 1:50. Serum dilution yielding 0.100 net $\mathrm{OD}$ value was considered as the RBD-binding serum titer.

\section{T-cell response}

Peripheral blood mononuclear cells (PBMC) were isolated from heparin-treated blood by standard density gradient centrifugation. The number of IFNy-producing spot forming cells (SFC) was determined by ELISpot as previously reported [12]. Briefly, PBMC $\left(2 \times 10^{5} / 100 \mu\right.$ l culture medium per well) were stimulated in duplicate for $24 \mathrm{~h}$ in 96 -well plates (coated with anti-IFN-y monoclonal capture antibody) with peptide pools (15mers, overlapping by 10 aminoacids, Pepscan, Lelystad The Netherlands) representative of the $S$ and peptide pool (15 mers, overlapping 11 aminoacids; JPT Peptide Technologies GmbH, Germany) 
representative of Nucleocapsid $(\mathrm{N})$ proteins, at the final concentration of $0.25 \mu \mathrm{g} / \mathrm{ml}$.

Phytoheamagglutinin (PHA; $5 \mu \mathrm{g} / \mathrm{mL}$ ) was used as positive control, and medium alone as negative control. For the evaluation of cell-mediated response against the SARS-CoV-2 variants, supernatant of

Vero E6 cells cultured in $25 \mathrm{~cm}^{2}$ flasks and infected for $72 \mathrm{~h}$ with $100 \mathrm{TCID}_{50} / \mathrm{ml}$ of different SARS-CoV-2 strains was UV-inactivated for 1 hour and used as antigen formulation. PBMC $\left(4 \times 10^{5} / 100 \mu \mathrm{l}\right.$ culture medium per well) were cultured in duplicate in the presence of inactivated SARS-CoV-2-infected (or mockinfected as negative control) cell supernatant diluted 1:10 in culture medium. Culture medium was RPMI 1640 supplemented with $2 \mathrm{mM} \mathrm{L-glutamine,} 100 \mathrm{U} / \mathrm{ml}$ penicillin and $100 \mu \mathrm{g} / \mathrm{ml}$ streptomycin, and $10 \%$ of heat inactivated fetal bovine serum (FBS, Euroclone). Spots were counted using an automated AID ELISPOT reader system (Autolmmun Diagnostika GmbH, Strasburg, Germany). The mean number of spots from duplicate cultures were adjusted to $1 \times 10^{6} \mathrm{PBMC}$. The net spots per million PBMC was calculated by subtracting the number of spots in response to negative control from the number of spots in response to the $S$ or $\mathrm{N}$ antigen. Responses $\geq 10$ net spots/million PBMC were considered positive based on background results obtained with negative control (mean SFC+2SD).

\section{Characterization of $C D 4^{+}, C D 4^{+}$follicular helper $\left(T_{F H}\right)$, and $C D 8^{+}$T-cell proliferative response}

To evaluate T-cell subsets proliferation, PBMC $(600,000 / 200 \mu l$ culture medium per well) collected from 19 vaccinated subjects, 9 subjects who experienced mild SARS-CoV-2 infection and 5 unexposed control subjects were stimulated in triplicate in 96-well round-bottom plates with peptide pools representative of the $S$ and N proteins, at the final concentration of $0.1 \mu \mathrm{g} / \mathrm{ml}$ for 7 days. Peptide pool from human actin, was used as negative control antigen. Culture medium was RPMI 1640 supplemented with $2 \mathrm{mM} \mathrm{L-}$ glutamine, $100 \mathrm{U} / \mathrm{ml}$ penicillin and $100 \mu \mathrm{g} / \mathrm{ml}$ streptomycin, $5 \%$ of heat inactivated human serum $A B, 1$ $\mathrm{mM}$ Sodium Pyruvate, $100 \mu \mathrm{M}$ non-essential amino acids and 50 $\mu \mathrm{M}$ 2-Mercaptoethanol. After culture, cells were washed with PBS 0,5 $\mu$ M EDTA and stained in PBS with Live/Dead Fixable Violet Dye (Invitrogen) at $4^{\circ} \mathrm{C}$. After washing, cells were stained at room temperature in PBS $5 \%$ FCS with antiCXCR5, followed by anti-lgG2b (biotinylated) and, subsequently, with Streptavidin BV421, CD3 PerCP 5.5, CD4 APC Cy7, CD8 FITC, CD25 PECy7, CD278 (ICOS) APC antibodies. Finally, cells were washed and suspended in $1 \%$ paraformaldehyde. The frequency of $\mathrm{CD}_{2} 5^{+} \mathrm{ICOS}^{+}$expanded $\mathrm{CD} 3^{+} \mathrm{CD} 4^{+}$, $\mathrm{CD}^{+}{ }^{+} \mathrm{CD} 4^{+} \mathrm{CXCR}^{+}$and $\mathrm{CD} 3^{+} \mathrm{CD} 8^{+}$T-cells was determined by subtracting the frequency of PBMC incubated with actin peptides from the frequency of PBMC incubated with SARS-CoV-2 $S$ and $N$ peptides. Flow-cytometry analyses were performed with a FACS Canto II flow cytometer and DIVA software (BD Biosciences). A representative pseudocolor plot analysis is shown in Supplementary figure 2.

\section{Antibody response}

Antibody response was determined using the chemiluminescent assay Elecsys Anti-SARS-CoV-2 S (Roche Diagnostics Rotkreuz, Switzerland), which provides quantitative measures of mainly lgG (but also IgA and IgM) specific for SARS-CoV-2 RBD. Results are given as units (U)/ml and are considered positive when $\geq 0.8 \mathrm{U} / \mathrm{ml}$. Neutralizing antibody serum titre was determined as previously reported [39]. Results were considered positive if higher or equal to 1:10 serum titre. 


\section{Statistical analysis}

Percentages with the $95 \%$ confidence interval $(95 \% \mathrm{Cl})$, median, range and interquartile range were reported. Levels of SARS-CoV-2-specific iCammunological parameters detected at T1 and T2 in naive subjects was performed with the Wilcoxon matched paired test, whereas levels of SARS-CoV-2-specific immunological parameters detected at $\mathrm{T} 0, \mathrm{~T} 1$ and $\mathrm{T} 2$ in experienced subjects were compared with the Friedman test for repeated measures (with correction for multiple comparisons and Dunn's post-test). Number of lymphocytes subpopulations at T0, T1 and T2 were given as mean number/ $\mu \mathrm{L} \pm \mathrm{SD}$ and compared with ANOVA for repeated measures (with correction for multiple comparisons and Tuckey posttest). Two-group unpaired data were compared with the Mann-Whitney U-test. Spearman's correlation (and $95 \% \mathrm{Cl}$ ) between the different immunological assays, and between immune response and subjects age were calculated at each time point analyzed. All analyses will be performed using GraphPad 8.3.0

\section{Declarations}

\section{Acknowledgments}

This work was supported by Fondazione Cariplo [grant CoVIM, no. 2020-1374] and Ministero della Salute, Ricerca Finalizzata [grant BIAS no. 2020-12371760] and Ricerca Corrente, [grant no. 80206] and from European Commission - Horizon 2020 [EU project 101003650 - ATAC]

\section{Author contribution}

IC and DL analyzed and interpreted the data and drafted the manuscript; IC, FeB and EG collected and managed the data; FeB, EG, KMGA, FZ, GC, performed experiments on T-cell response; EP, JCS, AF, PZ, AS performed experiments on antibody response; EP performed virus isolation and production; $A P$ sequenced viral genomes; AR, VZ enrolled the participants; FM and FN collected samples with SARS-CoV2 variants; $L S$ and LV produced recombinant RBD; DL wrote the protocol; FaB supervised the project and revised the manuscript. All the authors critically reviewed the manuscripts.

\section{Competing interests}

The authors have no competing interest to declare.

\section{References}

1. Walsh, E.E. et al. Safety and Immunogenicity of Two RNA-Based Covid-19 Vaccine Candidates. $N$ Engl J Med. 383(25), 2439-2450 (2020).

2. Polack, F.P.et al. Safety and Efficacy of the BNT162b2 mRNA Covid-19 Vaccine. N Engl J Med. 383(27), 2603-2615 (2020)

3. Baden, L.R. et al. Efficacy and Safety of the mRNA-1273 SARS-CoV-2 Vaccine. N Engl J Med. 382(19), 1851-1852 (2021). 
4. Sahin, U. et al. COVID-19 vaccine BNT162b1 elicits human antibody and $T(H) 1 T$ cell responses. Nature. 585(7823), 107-112 (2020).

5. Wang, Z. et al. mRNA vaccine-elicited antibodies to SARS-CoV-2 and circulating variants. Nature. Online ahead of print (2021).

6. Abu Jabal, K. et al. Impact of age, ethnicity, sex and prior infection status on immunogenicity following a single dose of the BNT162b2 mRNA COVID-19 vaccine: real-world evidence from healthcare workers, Israel, December 2020 to January 2021. Euro Surveill 26(6), 2100096 (2021).

7. Jackson, L.A. et al. mRNA-1273 Study Group. An mRNA Vaccine against SARS-CoV-2 - Preliminary Report. N Engl J Med. 383(20), 1920-1931 (2020).

8. Anderson, E.J. et al. Safety and Immunogenicity of SARS-CoV-2 mRNA-1273 Vaccine in Older Adults. N Engl J Med. 384(1), 80-82 (2020).

9. Tang, F. et al. Lack of peripheral memory B cell responses in recovered patients with severe acute respiratory syndrome: A six-year follow-up study. J Immunol. 186(12), 7264-8 (2011).

10. Dan JM, Mateus J, Kato Y, Hastie KM, Yu ED, Faliti CE, Grifoni A, Ramirez SI, Haupt S, Frazier A, Nakao C, Rayaprolu V, Rawlings SA, Peters B, Krammer F, Simon V, Saphire EO, Smith DM, Weiskopf D, Sette A, Crotty S. Immunological memory to SARS-CoV-2 assessed for up to 8 months after infection. Science 371(6529), eabf4063 (2021).

11. Sherina, N. et al. Persistence of SARS-CoV-2-specific B and T cell responses in convalescent COVID19 patients 6-8 months after the infection. Med (N Y) 2(3), 281-295.e4 (2021).

12. Cassaniti, I. et al. SARS-CoV-2 specific T cell immunity in COVID-19 convalescent patients and unexposed controls measured by ex-vivo ELISpot assay. Clin Microb Infect. Online ahead of print (2021).

13. Davies, N.G. et al. Estimated transmissibility and impact of SARS-CoV-2 lineage B.1.1.7 in England. Science. Online ahead of print (2021).

14. Voloch, C.M. Genomic characterization of a novel SARS-CoV-2 lineage from Rio de Janeiro, Brazil. J Virol. Online ahead of print (2021).

15. Tegally, H. et al. Sixteen novel lineages of SARS-CoV-2 in South Africa. Nat Med. 27(3), 440-446 (2021).

16. Xie, X. et al. Neutralization of N501Y mutant SARS-CoV-2 by BNT162b2 vaccine-elicited sera. bioRxiv01.07.425740 (2021).

17. Rees-Spear, C. et al. The effect of spike mutations on SARS-CoV-2 neutralization. Cell Rep. 34(12), 108890 (2021).

18. Madhi, S.A. et al. Efficacy of the ChAdOx1 nCoV-19 Covid-19 Vaccine against the B.1.351 Variant. $N$ Engl J Med. Online ahead of print (2021).

19. World Health Organization. Background document on the Janssen Ad26.COV2.S (COVID-19) vaccine: Background document to the WHO Interim recommendations for use of Ad26.COV2.S (COVID-19) 
vaccine https://www.who.int/publications/i/item/WHO-2019-nCoV-vaccines-SAGE-recommendationAd26.COV2.S-background-2021.1. Published 17 March 2021

20. John, S. et al. Multi-antigenic human cytomegalovirus mRNA vaccines that elicit potent humoral and cell-mediated immunity. Vaccine. 36(12), 1689-1699 (2018).

21. Manisty, C. et al. Antibody response to first BNT162b2 dose in previously SARS-CoV-2-infected individuals. 397(10279), 1057-1058 (2021).

22. Prendecki, M. et al., SARS-CoV-2 Antibody Point-of-Care Testing in Dialysis and Kidney Transplant Patients With COVID-19. Kidney Med. 3(1), 54-59.e1 (2021).

23. Bradley, T. et al. Antibody responses boosted in seropositive healthcare workers after single dose of SARS-CoV-2 mRNA vaccine. medRxiv02.03.21251078 (2021).

24. Robbiani, D.F. et al. Convergent antibody responses to SARS-CoV-2 in convalescent individuals. Nature 584:437-442 (2020).

25. Van Elslande, J. et al. Symptomatic SARS-CoV-2 reinfection by a phylogenetically distinct strain. Clinical Infectious Diseases. Clin Infect Dis ciaa1330 (2020).

26. Larson, D. et al. A case of early Re-infection with SARS-CoV-2. Clin Infect Dis. (2020)

27. Greaney, A.J. et al. Mutational escape from the polyclonal antibody response to SARS-CoV-2 infection is largely shaped by a single class of antibodies. bioRxiv. 2021.03.17.435863 (2021).

28. Weisblum, Y. et al. Escape from neutralizing antibodies by SARS-CoV-2 spike protein variants. ELife 9, e613122020 (2020).

29. Wibmer, C.K. et al. SARS-CoV-2 501Y.V2 escapes neutralization by South African COVID-19 donor plasma. bioRxiv01.18.427166 (2021).

30. Garcia-Beltran, W.F. et al. COVID-19-neutralizing antibodies predict disease severity and survival. Cell 184(2):476-488.e11 (2021).

31. Wang, P. et al. Antibody Resistance of SARS-CoV-2 Variants B.1.351 and B.1.1.7. Nature Online ahead of print (2021).

32. Tarke, A. et al. Negligible impact of SARS-CoV-2 variants on CD $4+$ and $C D 8+T$ cell reactivity in COVID-19 exposed donors and vaccines. BioRxiv02.27.433180 (2021)

33. Grifoni, A. et al. Targets of T Cell Responses to SARS-CoV-2 Coronavirus in Humans with COVID-19 Disease and Unexposed Individuals. Cell 181(7), 1489-1501.e15 (2020).

34. Sette, A \& Crotty, S. Pre-existing immunity to SARS-CoV-2: the knowns and unknowns. Nat Rev Immuno/ 20(8), 457-458 (2020).

35. Le Bert, N. et al. SARS-CoV-2-specific T cell immunity in cases of COVID-19 and SARS, and uninfected controls. Nature 584(7821), 457-462 (2020).

36. Meckiff, B.J. et al. Single-cell transcriptomic analysis of SARS-CoV-2 reactive CD4 + T cells. bioRxiv06.12.148916 (2020).

37. Liu, M.A. A Comparison of Plasmid DNA and mRNA as Vaccine Technologies. Vaccines 7(2), 37 (2019). 
38. De Gasparo, R et al. Bispecific IgG neutralizes SARS-CoV-2 variants and prevents escape in mice. Nature. Online ahead of print (2021).

39. Percivalle, E et al. Prevalence of SARS-CoV-2 specific neutralising antibodies in blood donors from the Lodi Red Zone in Lombardy, Italy, as at 06 April 2020. Euro Surveill 25(24), 2001031 (2020).

\section{Figures}

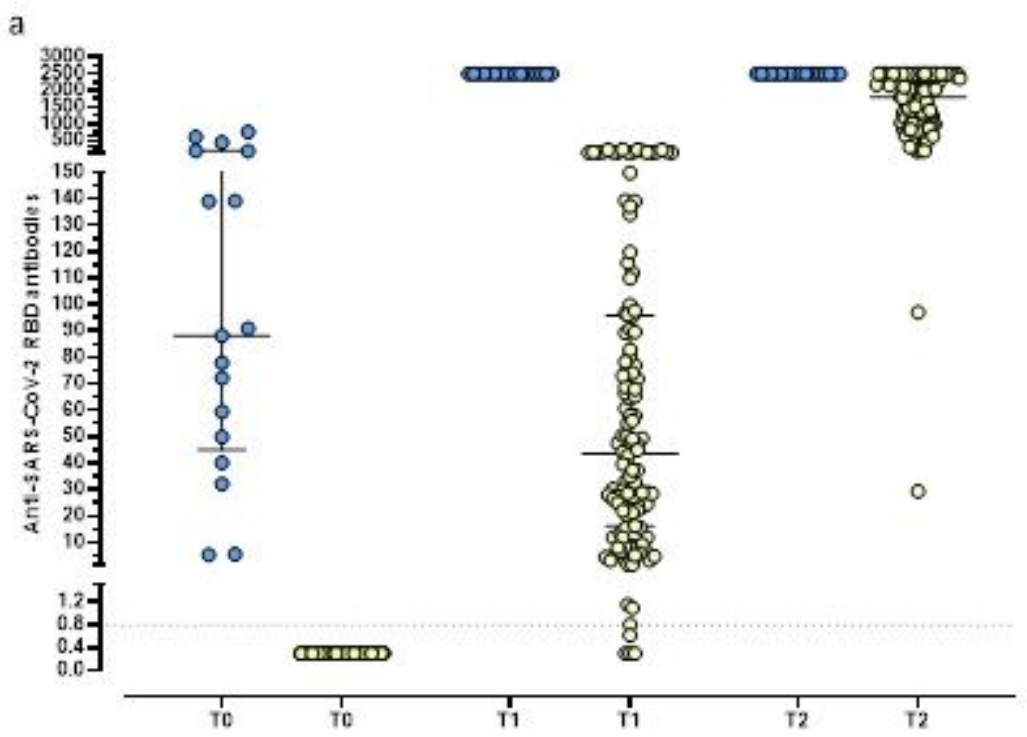

b

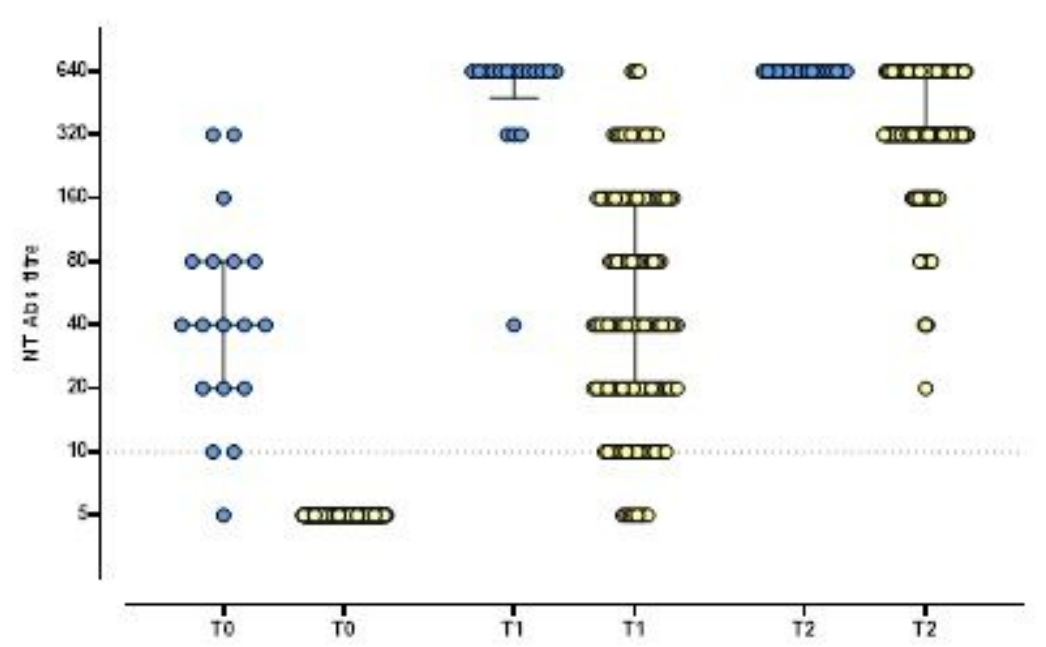

- SARS-COV-2 serapabltwe subjects O SARS-COV-2 seronegatwe subjects

\section{Figure 1}

Anti-SARS-CoV-2 RBD response measured by Elecsys Anti-SARS-CoV-2 S assay (A) and neutralizing (NT) antibodies anti-SARS-CoV-2 (B) measured at baseline (T0), day 21 (T1) and day 42 (T2) in 127 COVID-19 naive and 18 COVID-19 experienced subjects. The dotted horizontal line represents the positive level of responses. 


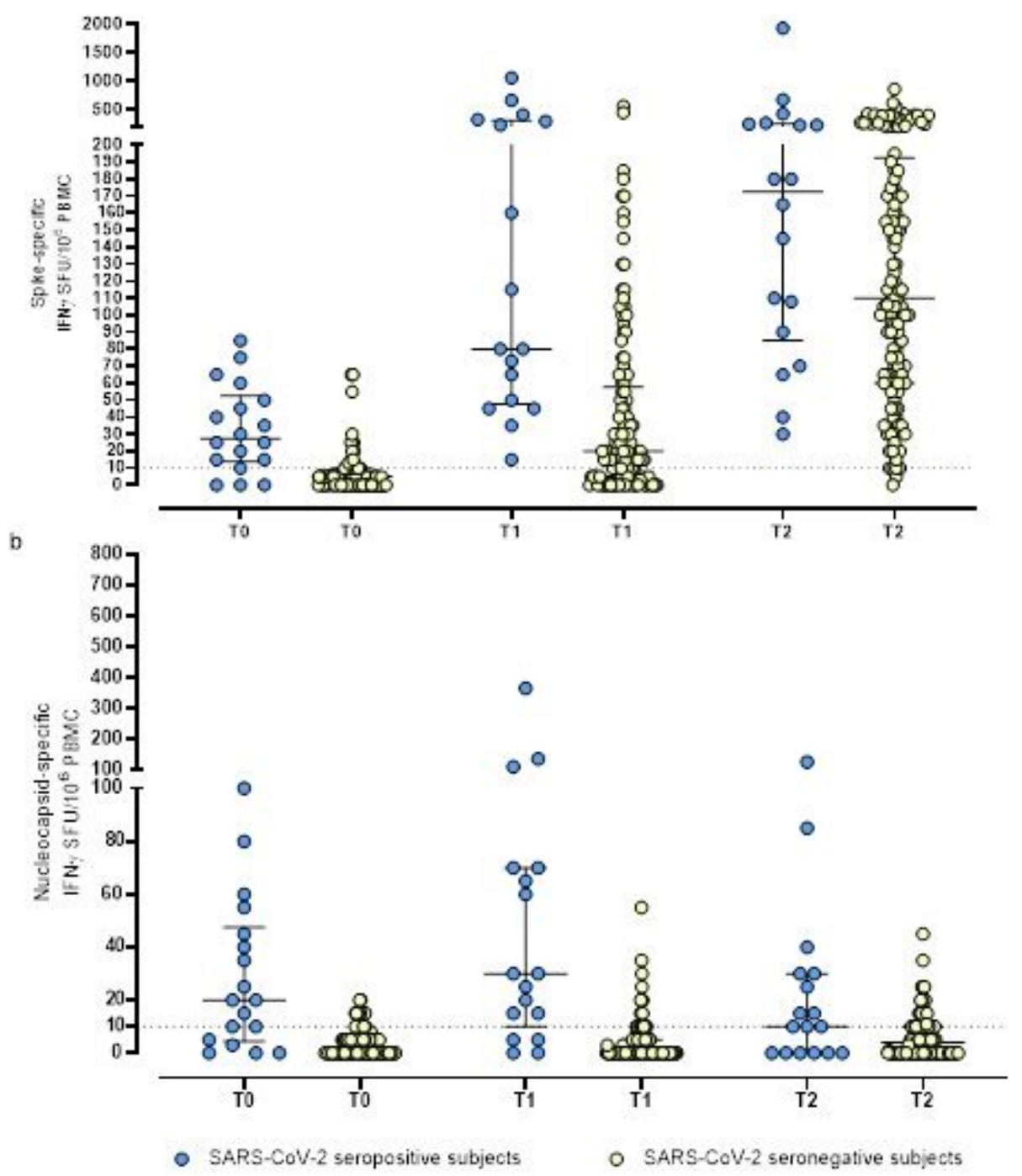

Figure 2

Cell mediated immune response against Spike $(\mathrm{S})$ and Nucleocapsid $(\mathrm{N})$ peptide pools in 145 vaccinated subjects (127 naïve for SARS-CoV-2 infection and 18 who experienced SARS-CoV-2 infection) at T0, T1 and T2. The dotted horizontal line represents a positive cut off for SARS-CoV-2 T-cell response.
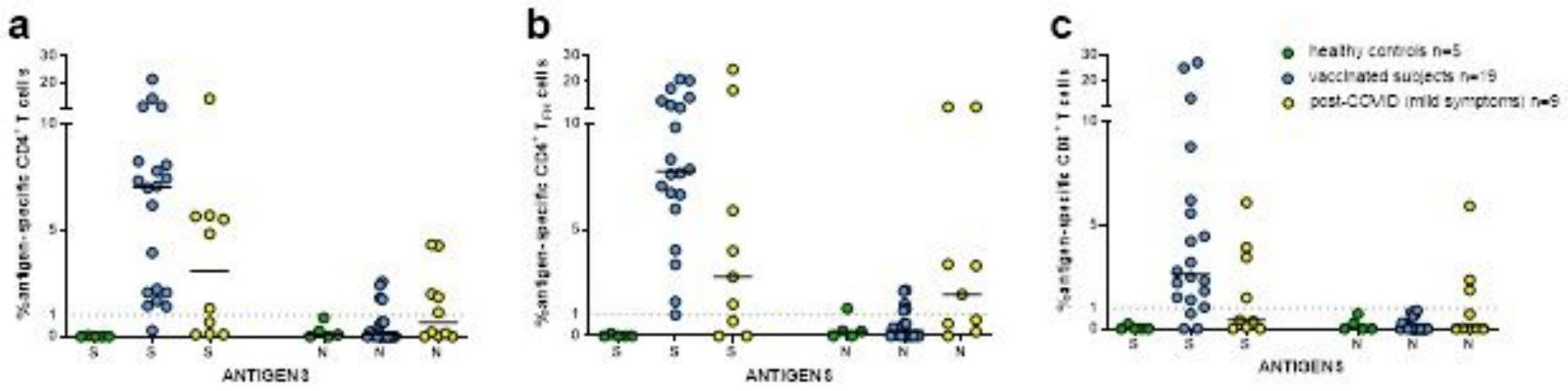

Figure 3 
CD4+ T (a), CD4+ TFH (b) and CD8+ T (c) cells proliferating in response to Spike (S) and Nucleocapsid $(\mathrm{N})$ protein peptide pools of SARS-CoV-2 in healthy controls, vaccinated subjects tested after the complete vaccination schedule, and post-COVID subjects with mild symptoms tested a median time of 38 (range 28-90) days after infection. The percentage of activated CD25+ICOS+ cells after 7-day stimulation is reported and black bars represent median values. Horizontal dotted lines indicate the cut-off for positive response.
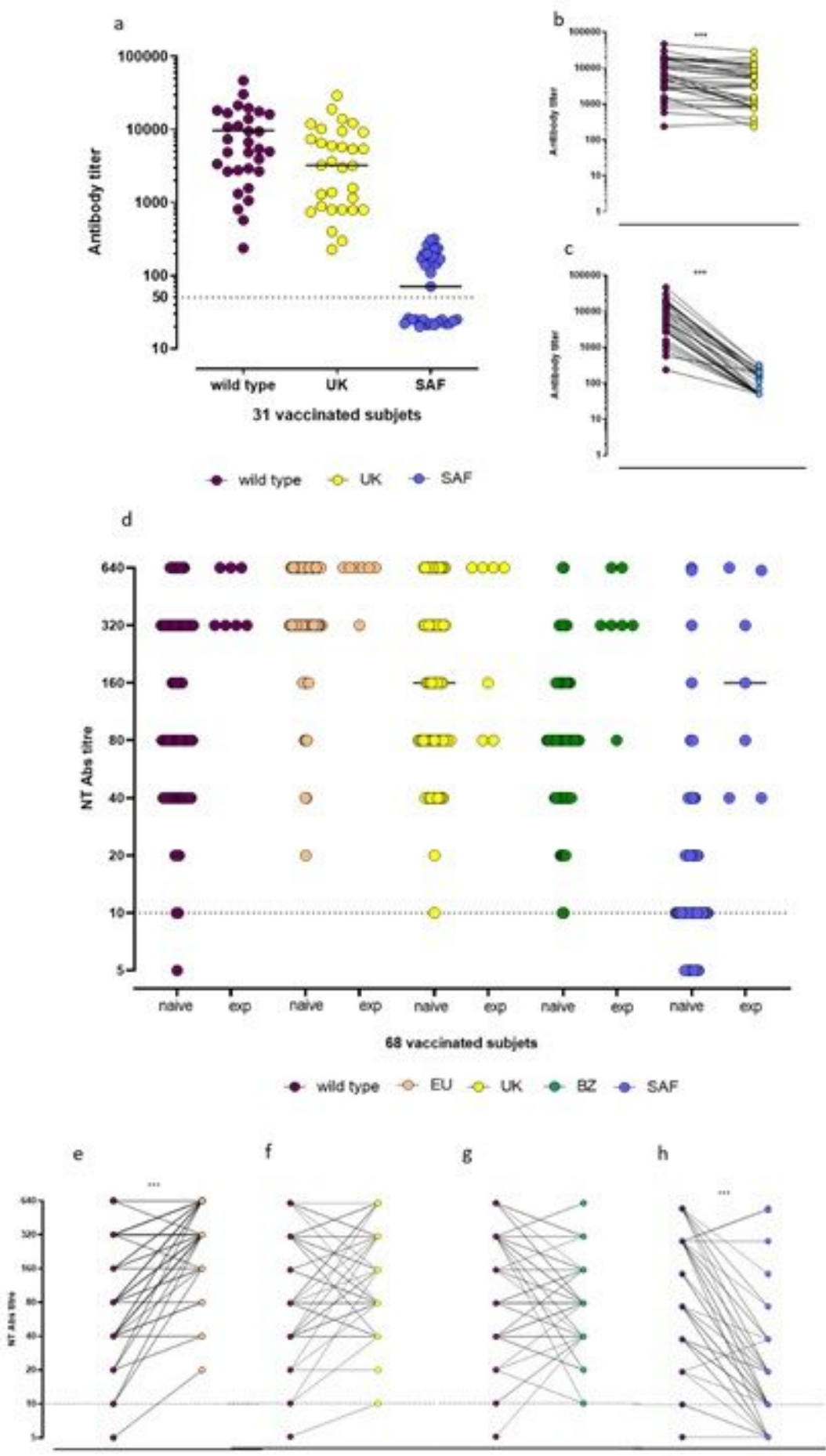

Figure 4 
Median RBD-specific antibody levels against wild type, UK and SAF strains are shown (a). Comparison between wild type vs the SAF (b) and between wild type vs the UK (c) RBD-specific antibody titres was made. SARS-CoV-2 NT Abs against all the variants have been measured in 68 vaccinated subjects (61 were SARS-CoV-2 naïve and 7 experienced SARS-CoV-2 infection; naïve vs exp) (d). Comparison between wild type vs the EU (e), wild type vs the UK (f), wild type vs the BZ $(\mathrm{g})$ and wild type vs the SAF (h) have been shown: $(* \star *) p$ value $<0.001$.

a

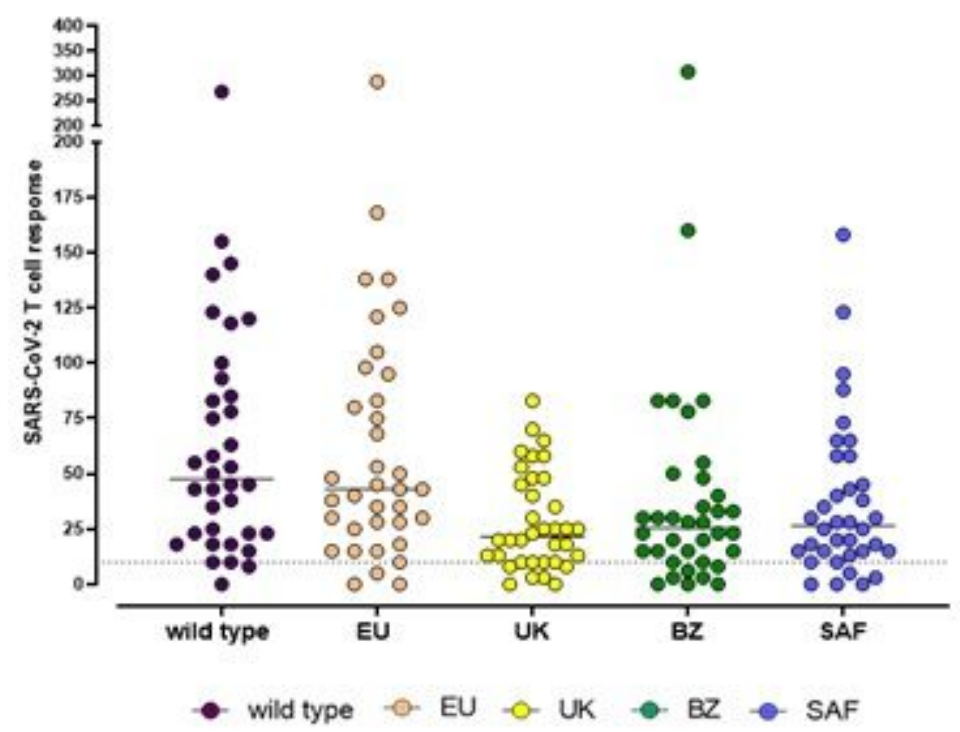

b

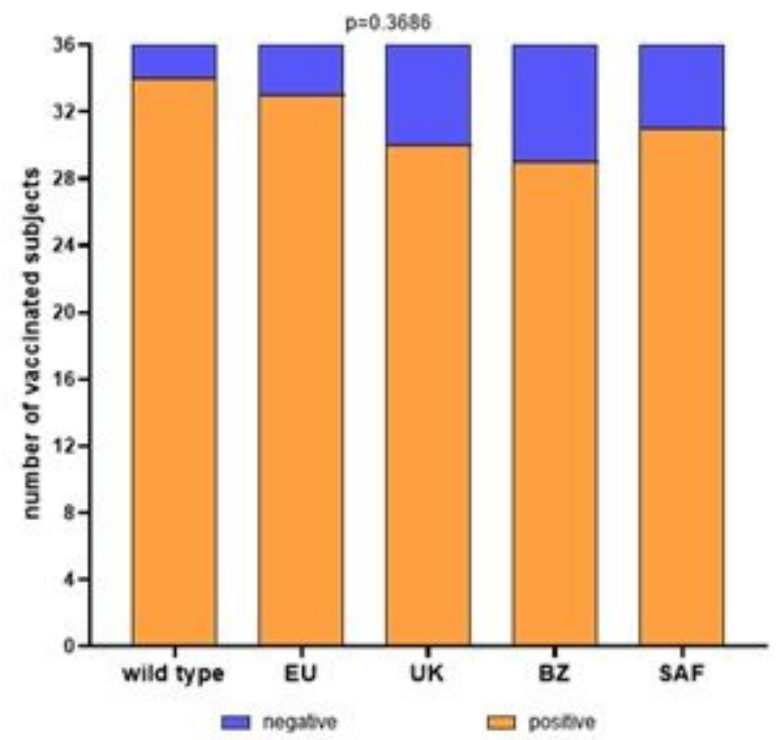

Figure 5

Median T cell response against SARS-CoV-2 variants are shown in section a, while number of subjects with positive SARS-CoV-2 T-cell response and negative SARS-CoV-2 T-cell response is shown in section b. 


\section{Supplementary Files}

This is a list of supplementary files associated with this preprint. Click to download.

- flatLilleriepc.pdf

- flatLillerirs.pdf

- SupplementaryFigures.pdf 